\title{
S52. Assessment of treatment of psychiatric disorders
}

\section{ANXIETY IN DEPRESSION: ASSESSMENT IN CONTROLLED CUINICAL TRIALS}

EO Salinas', JD Guelfi'.

1: Wyeth-Ayerst European CR\&D, 6 rue Clisson, 75013 PARIS.

2: Clinique des Maladies Mentales et de l'Encéphale, 100 rue de la Santé, 75014 PARIS.

The classical model of Kielholz propose that antidepressants are active on three components of the depressive syndrome: depressed mood, motor retardation and anxiety. According to this model, antidepressants can be sub-divided into activating versus sedativeanxiolytic compounds. Although during clinical trials in major depression the course of anxiety symptoms during treatement are usually evaluated, the complexity of the relationship between anxiety and depression can not be fully assessed only in terms of the improvement of a cluster of symptoms.

This report focus on the theoretical basis and the methodology for the assessment of anxiety as a part of the depressive syndrome, from the perspective of the clinical development of new drugs with putative antidepressant properties.

At least three basic questions of clinical relevance should be addressed in order to evaluate the anxiolytic profile of a new antidepressant:

- are anxiety symptoms in the sample secondary to the depressive state? (i. e.: improvement of core depressive symptoms determines relief of anxiety irrespective of the anxiolytic or activating profile of the drug?)

- what is the prognostic value of anxiety at baseline with respect to outcome with a particular drug?

- what is the part of drug-induced anxiety symptoms in the overall outcome of anxiety?

Different methodological strategies are proposed to address this questions.
THE CHOICE OF THE PRIMARY EFFICACY-CRITERIA AND OF THE TYPE OF STATISTICAL ANALYSIS IN ANTIDEPRESSANT CLINICAL TRIALS.

P Boyer

INSERM, CMME, 100 rue de la Santé, 75014, Paris, France.

The main primary criteria of efficacy used in most of the antidepressant clinical trials conducted in the ten last years are as follows:

- Hamilton depression rating scale (HAMD 17 items): change from baseline to last visit in total score; percentage change from baseline to last visit in total score; change from baseline in item I (depressed mood).

-Clinical global impression (CGl): change from baseline in severity of illness score; global improvement score compared to entrance to study. Responder analysis: percentage of patients having achieved at least $50 \%$ improvement in HAMD total score and a 1 or 2 score at the CGI global improvement score at last visit.

The choice of other primary criteria is two-to-tentold less frequent than the choice of those mentioned above. Based on those criteria the intent to treat analysis is the most common statistical approach and concerns all the randomized patients as well as the evaluable patients with the last observation carried forward. As a rule parametric tests are used for HAMD global scores; non parametric tests are used for isolated Items and the CGI.

If such analyses allow to draw calcar conclusions on the efficacy of a drug, some aspects of the change induced by antidepressant treatments are not sufficiently taken into account. The better knowledge of the time course and pattern of changes and of the quality and the maintenance of the response necessitate to investigate more specific models such as: stratification and subtyping according to initial characteristics of the patients (severity, etc. time point, pattem and sustained responder analyses as well as different types of cluster analyses. 
ANTIMANIC AND PROPHYLACTIC PROPERTIES OF CARBAMAZEPINE IN AFFECTIVE DISORDERS : SOME METHODOLOGICAL CONSIDERATIONS. R. Dardennes, C. Even, F. Bange, A. Heim. Clinique des Maladies Mentales et de l'Encéphale, Centre Hospitalier Sainte-Anne, 1, rue Cabanis, 75014 Paris, France.

During the eighties, Carbamazepine (CBZ) has gained the leading position as an alternative treatment for mania and the prophylaxis of bipolar disorders. The purpose of this work was to examine, by means of meta-analysis, the validity of the opinions stating that $\mathrm{CBZ}$ equals lithium in terms of prophylactic efficacy and that $\mathrm{CBZ}$ has an equivalent antimanic efficacy to neuroleptics and lithium. Separate meta-analyses were conducted and only considered controlled studies. In both cases, lack of statistical power questions the conclusion of an "equivalence" between CBZ and drugs it has been compared to.

\section{EVALUATING TREATMENT FOR PSYCHIATRIC DISORDER IN GENERAL CARE: THE COCHRANE COLLABORATION \\ $M$ G Gelder}

University Department of Psychiatry, Warneford Hospital, Oxford, OX3 7JX, UK

The Cochrane Collaboration has been developed to produce systematic up to date reviews of all randomised clinical trials of health care. The work began in Oxford with reviews of randomised controlled trials of treatment in pregnancy and childbirth and the value of this work for clinical practice has prompted its extension to other specialties of medicine. Cochrane Centres have been set up in several countries other than the United Kingdom of which Denmark is one.

In each subject an editorial team collects and assesses all the controlled clinical trials carried out worldwide, searching journals and conference reports in many languages. Studies of psychiatric journal articles have shown that existing data bases cannot be relied on to assemble material needed for a systematic review and special methods of enquiry have to be adopted. For each treatment this edited material is reviewed critically by a group of experts. The results of this review are updated continuously as new information is added to the data base. A collaborative group is already working on clinical trials in schizophrenia and other collaborations in the area of psychiatry are being established including one on psychiatric disorders in general care.
APPLICATIONS OF LATENT TRAIT ANALYSIS (LTA) IN PSYCHIATRY : A REVIEW OF THE LITERATURE Ph Cialdella (Lyon, France). M. De Bonis (Paris, France), P. Bech (Hillerod. Denmark)

Service de Pharmacologie Clinique (Pr J.P. Boissel) B.P. 3041 Lyon Cedex 03, France.

LTA is a family of probabilistic methods (the simplest being the Rasch model) used to model the probability for a subject, to respond to an item, depending on the relative positions of the subject and the item on a latent unobserved dimension (rait).

We have found 31 published studies, of which 23 were conducted in Europe, principally in Denmark (13 studies). Each study had to include at least some subjects suffering from a psychiatric disorder, and to use a LTA method.

The instruments were mainly observer scales; the principal domain explored was depression, and/or associated dimensions such as endogeneity/reactivity, or melancholia. Other dimensions explored were : anxiety, psychological distress, psychosis, behaviour disorders, etc. The different softwares used, can be classified according to their ability to cope with multicategory items (Likert scales), to perform 2-parameter LTA, and to test for several dimensions. The Rasch model was the most frequently used method ( 24 studies). Global tests of fit of the model were given in 29 studies, item parameter values in 14 studies, itern-specific tests in 9 studies; other output like subject's parameter values, Item Characteristic Curves (ICC) were rarely given.

Methodological weaknesses included small number of subjects ( $1 / 3$ of the studies), distributions of subjects on severity of illness (Latent Trait) much homogeneous, lack of information on the selection of items fullfilling the model, use of too simple and restrictive models (Rasch models), incomplete testing of multidimensional hypotheses, etc. In addition, some psychopathological phenomena do not seem to follow a latent trait structure.

In conclusion, efforts should be made to explain the usefulness of LTA (compared with factor analysis) to psychiatrists, and to expand its application in order to improve the psychometric properties of measurement instruments in psychiatry and psychopathology.

CHANGES IN ACUTE PSYCHIATRIC WARDS: AN EXAMPLE OF MONITORING TREATMENT PROGRAMS BY THE USE OF ROUTINE DATA.

Friis, S. Department of psychiatry. Ullevål University Hospital. N-0407 oslo Norway.

objectives: 1 . To demonstrate how routinely collected data can monitor health care delivery systems. 2. To show how the therapeutic challenges have changed in acute psychiatric wards.

Material and methods: From 1981 all psychiatric wards i os10, Norway have registered on a routi-ne basis several data concerning every admission. This paper will present the annual numbers for three acute wards covering a catchment area of 300000 people.

Results: The number of admissions was basically unchanged during the period. There was a marked increa-se in the percentage of emergencies, admittances due to psychosis and committed admissions. Mean length of stay ranged from 18 to 40 days at the start of the period, but was gradualiy reduced to $<20$ days for all the wards. Conclusion: The routine data clearly demonstrated that during the 80 's there was a dramatically increased burden on the acute wards in oslo. 